\title{
Navegación en interiores utilizando la tecnología beacons
}

\author{
Alicia Martínez-Rebollar ${ }^{1}$, Luis Antonio López-García ${ }^{1}$, Hugo Estrada-Esquivel ${ }^{2}$, \\ Manuel Mejía Lavalle ${ }^{1}$, Javier Ortiz Hernández ${ }^{1}$ \\ ${ }^{1}$ Tecnológico Nacional de México/CENIDET, Cuernavaca, Morelos, México \\ ${ }^{2}$ Consejo Nacional de Ciencia y Tecnología, México \\ \{luis.lopez17ce@cenidet.edu.mx, amartinez, \\ mlavalle, ortiz\}@cenidet.edu.mx, hugo.estrada@conacyt.mx
}

\begin{abstract}
Resumen. El internet de las cosas (IoT por sus siglas en inglés) está impulsando la generación de edificios inteligentes. Estos edificios tienen como requisito principal la navegación de interiores. Sin embargo, la tecnología GPS, la cual es utilizada para llevar a cabo el posicionamiento, no es posible utilizarla dentro de un edificio debido a que las señales satelitales no viajan bien a través de materiales sólidos. De esta manera, se han propuesto sensores, dispositivos Bluetooth, o RFID que se utilizan en los edificios inteligentes. En este artículo presentamos una propuesta de navegación de interiores, la cual utiliza la tecnología beacons y los teléfonos inteligentes. Nuestra aplicación de software obtiene información del contexto y genera la mejor ruta para llegar al destino dentro de un edificio inteligente. Se utilizó el algoritmo Dijkstra para procesar toda la información. De ahí que nuestra propuesta tiene como objetivo combinar diferentes tecnologías, y adaptar algoritmos desarrollados a la navegación de interiores.
\end{abstract}

Palabras clave: navegación de interiores, tecnología beacons, edificio inteligente, internet de las cosas.

\section{Indoor Navigation using Beacon Technology}

\begin{abstract}
The Internet of Things (IoT) is promoting the generation of smart buildings. These buildings have as main requirement the navigation of interiors. However, GPS technology, which is used to carry out positioning, it cannot be used within a building because satellite signals do not travel well through solid materials. In this way, sensors, Bluetooth devices, or RFID that are used in smart buildings have been proposed. In this paper, we present an interior navigation proposal, which uses beacons technology and smartphones. Our software application obtains information from the context and generates the best route to reach the destination within an intelligent building. The Dijkstra algorithm was used to process all the information. Hence, our proposal aims to combine different technologies, and adapt developed algorithms to indoor navigation.
\end{abstract}

Keywords: navigation indoor, beacons technology, smart building, internet of things. 


\section{Introducción}

En las últimas décadas, la disponibilidad de dispositivos móviles con el Sistema de Posicionamiento Global (GPS) ha impulsado el desarrollo de servicios y aplicaciones basados en la ubicación. Entre los más populares están las aplicaciones de navegación que proporcionan contextos y son instrucciones para guiar a sus usuarios. Sin embargo, dado que el GPS requiere una línea de visión sin obstrucciones entre el receptor de GPS y múltiples satélites, su disponibilidad en ambientes interiores típicos está severamente limitada. Como resultado, la mayoría de las aplicaciones de navegación de amplio espectro se centran en escenarios al aire libre o proporcionan solo soporte para entornos interiores [1].

Para proporcionar una alternativa al GPS, los investigadores y profesionales han desarrollado diversos enfoques para la localización en interiores que aprovechan la visión, el sonido, la tecnología de radiación infrarroja o radiofrecuencia, por nombrar solo algunos. Al usar estos sistemas, muestran claramente que es posible proporcionar información precisa sobre la ubicación a un costo razonable. Sin embargo, la mayoría de las aplicaciones en este campo se enfocan en escenarios interiores aislados. Como consecuencia, sólo brindan soporte en un ambiente interior particular, como un centro comercial o un edificio universitario, por lo tanto, no pueden proporcionar una verdadera experiencia de navegación de extremo a extremo [2].

Otro aspecto el cual se debe de tomar en cuenta es el tiempo, es decir vivimos constantemente en un proceso de cambio. Según la ONU el 54 por ciento de la población mundial actual reside en áreas urbanas y se prevé que para 2050 llegará al 66 por ciento [3]. Actualmente se está viviendo la transformación de las ciudades en ciudades inteligentes. Para preparar un proyecto de ciudades inteligentes se requiere de diferentes etapas, retos, un diagnóstico previo de los problemas de la administración pública y de la ciudad y una evaluación de las oportunidades. El diseño de una solución inteligente requiere identificar los recursos tecnológicos que se necesitan para el desarrollo de proyectos.

Una ciudad se vuelve más eficiente en la medida en que es capaz de obtener datos generados en el ambiente, en las infraestructuras instaladas por prestadores de servicios $\mathrm{y}$ ciudadanos en los edificios y en las calles para luego procesar esos datos y transformarlos en información que facilite la toma de decisiones capaces de mitigar, organizar, anticipar o prever innumerables retos urbanos. Para captar esos datos, en algunos casos es necesario instalar sensores y cámaras de video en la infraestructura física de la ciudad, conectarlos entre sí en una red de comunicación de datos, para luego usar esos datos enviados en tiempo real y apoyar de esa manera a la toma de decisiones [3].

Cisco estima que el universo de "Internet de las Cosas" (IoT) en 2020 contará con 50.000 millones de dispositivos [4].

La navegación de interiores es una gran tendencia que se encuentra ya vigente en las grandes ciudades. Se ha dado un gran salto de la navegación en exteriores a la navegación en interiores, teniendo la necesidad de localizar lugares y objetos en espacios cerrados.

De esta manera, nuestra propuesta tiene como objetivo combinar diferentes tecnologías como: beacons y teléfonos inteligentes para la navegación de interiores, extendiendo el algoritmo Dijkstra para llevar a cabo la navegación. 
Esté artículo se encuentra organizado de la siguiente manera: en la Sección 2 se presentan los fundamentos teóricos y trabajos relacionados. En la sección 3 se detalla nuestra propuesta de navegación en interiores y exteriores. La sección 4 muestra la manera en cómo estamos probando nuestra propuesta de navegación. Finalmente, la sección 5 se presentan las conclusiones y trabajos futuros de nuestra investigación.

\section{Fundamentos teóricos y trabajos relacionados}

En esta sección se detallan los fundamentos teóricos y trabajos relacionados en navegación de interiores y exteriores, utilizando diferentes tecnologías.

\subsection{Marco teórico}

Algunos conceptos relevantes en este trabajo de investigación se presentan a continuación.

Sistema de Posicionamiento Global. El Sistema de Posicionamiento Global o GPS es un sistema global de navegación por satélite que permite determinar en todo el mundo la posición de un objeto, una persona, un vehículo o una nave. Se puede alcanzar una precisión hasta de centímetros, usando el GPS diferencial, pero lo habitual son unos pocos metros.

El GPS funciona mediante una red de 27 satélites ( 24 operativos y 3 de respaldo) en órbita a $20.200 \mathrm{~km}$ sobre el globo terráqueo, con trayectorias sincronizadas para cubrir toda la superficie de la Tierra. Cuando se desea determinar una posición, el receptor que se utiliza para ello localiza automáticamente como mínimo tres satélites de la red, de los que recibe unas señales indicando la posición y el reloj de cada uno de ellos. Con base en estas señales, el aparato sincroniza el reloj del Sistema de Posicionamiento y calcula el retraso de las señales; es decir, la distancia al satélite. Por "triangulación" los tres satélites calculan la posición en que el GPS se encuentra. La triangulación en el caso del GPS se basa en determinar la distancia de cada satélite respecto al punto de medición. Conocidas las distancias, se determina fácilmente la propia posición relativa respecto a los tres satélites. Conociendo además las coordenadas o posición de cada uno de ellos por la señal que emiten, se obtiene la posición absoluta o coordenadas reales del punto de medición [5].

Navegación en interiores. La navegación en interior como la palabra lo indica navegar dentro de los edificios. Debido a que la recepción del GPS normalmente no existe dentro de los edificios, aquí se usan otras tecnologías de posicionamiento cuando se desea el posicionamiento automático. A menudo se utilizan Wi-Fi o beacons (Bluetooth Low Energy, BLE) para crear un llamado "GPS interior". La mayoría de las aplicaciones requieren una funcionalidad de "enrutamiento interior" que guía a las personas de forma precisa a través de un edificio utilizando una aplicación de navegación interior y de esta manera determina automáticamente su posición, muy similar a los sistemas de navegación que usamos en nuestros automóviles [6]. 
Beacons. En esta propuesta de investigación se utiliza la tecnología de beacons, como medio de localización de puntos de interés en la navegación de interiores. Los beacons son transmisores diseñados para usar señales de transmisión de bluetooth de baja energía para atraer la atención a una ubicación específica. Estos transmisores son muy pequeños y se pueden colocar en paredes o pilares de lugares como tiendas, aeropuertos, restaurantes, apartamentos, oficinas, etc.

La tecnología de beacons o baliza se usa principalmente para detectar la posición de su identificador en espacios interiores donde es imposible ubicar los dispositivos con la ayuda de señales de GPS [7].

Los siguientes tres conceptos (Identificador único universal, major, minor) son atributos que tienen todos los beacons que utilizan el protocolo ibeacon, estos atributos fueron utilizados para hacer una primera ubicación del usuario sin la necesidad de utilizar las credenciales que la plataforma Estimote genera para el uso de los beacons.

Identificador único universal. Un UUID es un número de 128 bits utilizado para identificar de manera única algún objeto o entidad en Internet. Dependiendo de los mecanismos específicos utilizados, se garantiza que un UUID es diferente o, al menos, es muy probable que sea diferente de cualquier otro UUID [8].

Major. Los valores principales son valores enteros sin signo entre 1 y 65535 los cuales están destinados a identificar y distinguir un grupo; por ejemplo, a todos los beacons de un determinado piso o habitación de su sede se les podría asignar un valor principal único [9].

Minor. Los valores principales son valores enteros sin signo entre 1 y 65535 los cuales están destinados a identificar y distinguir a un individuo, por ejemplo, distinguir beacons específicas dentro de un grupo de beacons asignadas a un valor Mayor [9].

\subsection{Trabajos relacionados}

Esté trabajo de investigación [10] describe un método para determinar la ubicación de un dispositivo móvil, como una computadora portátil o un teléfono móvil, en un ambiente interior usando beacons bluetooth. Una ventaja importante de este método es que permite que el dispositivo móvil determine su ubicación mientras permanece de manera anónima. En un despliegue de beacons en un edificio, una de las tareas importantes es el diseño de la colocación de beacons.

La propagación de señales en entornos interiores son complejos, ya que son afectadas por factores como la estructura del edificio, conductos, propiedades de transmisión y reflexión de los materiales de construcción, muebles y la interferencia de otros dispositivos. Por lo tanto, el área desde donde se ve una beacon es muy irregular y no muy aproximado por modelos simples como los elipsoides.

Otro trabajo de investigación es [11], el cual presenta un marco de combinación de sensores de teléfonos inteligentes e iBeacons para una localización interior precisa. El Pedestrian Dead Reckoning (PDR) se puede aplicar para la localización mediante sensores de teléfonos inteligentes, pero desafortunadamente podría ir a la deriva por la poca distancia que abarca. Por lo tanto, iBeacons aprovecha para corregir esta desventaja del enfoque PDR. Los autores realizaron un filtro de señales para la 
corrección de la poca distancia que abarca y los experimentos que son presentados en este documento muestran una mejora significativa de la precisión de localización con la dispersión del despliegue de iBeacon en un área de $47.3 \mathrm{~m}$ x 15.9m. Además, dado que la precisión de la estimación del punto inicial es vital para el enfoque de PDR, evaluaron el desempeño de su enfoque propuesto con respecto al error de estimación del punto inicial. Los resultados subrayan la solidez de su enfoque propuesto para el error de estimación del punto inicial.

El siguiente trabajo de investigación presenta el desarrollo de un Sistema de Navegación en Interiores usando la tecnología NFC [12]. NFC es una tecnología de comunicación inalámbrica bidireccional de corto alcance. La comunicación se produce entre dos dispositivos cercanos a pocos centímetros. NFC en interiores es muy simple y fácil de usar, el usuario sólo necesita llevar y usar un dispositivo móvil habilitado para NFC. La aplicación de navegación interior debe ser OTA (Over the Air) precargada en la tarjeta inteligente. De lo que el usuario necesita simplemente toque la etiqueta URL (etiqueta NFC), que contiene la URL de la información del mapa interior justo antes de ingresar al edificio o al área. El mapa en el sitio web es OTA descargado al dispositivo móvil del usuario desde MapServer (es decir, un servidor web que contiene la información del mapa). La aplicación de navegación en el interior del teléfono móvil se inicia automáticamente y luego utiliza está información del mapa. A medida que el usuario selecciona el punto de destino de su viaje dentro del edificio, la aplicación de navegación interior proporciona la ruta óptima hacia su destino.

Tabla 1. Comparativa de Google maps indoor y nuestra propuesta: NavIndoor.

\begin{tabular}{|c|c|c|}
\hline & Google maps indoor & NavIndoor \\
\hline Tecnología & GPS & Bluetooth \\
\hline Visualización & Mapas & Indicaciones \\
\hline Intermediarios & $\mathrm{Si}$ & No \\
\hline Disponibilidad & $\begin{array}{l}\text { Sólo está disponible para } \\
\text { algunas ciudades }\end{array}$ & Para todas las ciudades \\
\hline Tipo de público & $\begin{array}{c}\text { Centros comerciales y socios } \\
\text { existentes }\end{array}$ & Todo el público \\
\hline
\end{tabular}

El siguiente trabajo de investigación presenta el desarrollo de un sistema de navegación en interiores usando la tecnología GPS [13], el cual fue desarrollado por la empresa Google, este sistema de navegación está incorporado a la aplicación móvil de Google maps que ya es actualmente utilizada por la mayoría de la gente para buscar lugares o nos ayude a llegar a un destino. Esta aplicación funciona con solo acercarse al edificio, una vez dado el primer paso se muestra un mapa del edificio donde se puede observar aquellos lugares de interés, navegar a través del edificio. Sin embargo, la aplicación solo se encuentra enfocada a una específica parte de la población como socios y centros comerciales. En la tabla 1 se muestran las características de la aplicación de google maps indoor y la propuesta en este trabajo de investigación.

El siguiente trabajo de investigación presenta el desarrollo de un sistema de navegación en interiores usando la tecnología GPS [14], este sistema de navegación en interiores es llamado FSKTM 2D basado en Android usando el algoritmo A*, es una aplicación para Android para los estudiantes y visitantes de la Universidad Tun Hussein 
Onn Malasia. Esta aplicación de navegación se utiliza para encontrar la ubicación del aula por parte de los estudiantes. Permite a los estudiantes elegir su destino (el aula) y proporcionar el mapa de ruta a los estudiantes. Calcula el camino más corto para los estudiantes y los lleva a su destino.

\section{Propuesta de navegación de interiores: navIndoor}

La navegación en interiores es el siguiente paso en la localización después del GPS. Este tipo de navegación se ocupa de encontrar lugares y objetos en espacios cerrados como edificios, en donde las señales satelitales GPS no llegan con precisión.

Se utilizan en este tipo de navegación tecnologías como Wi-Fi o Balizas (Bluetooth Low Energy, BLE) para crear el llamado "GPS de interior". Este tipo de navegación interior permite guiar a las personas a navegar en edificios $y$ determinar automáticamente su posición, muy similar a los sistemas de navegación que se utiliza en los automóviles.

En este artículo se presenta nuestra propuesta de investigación sobre navegación de interiores, en la cual se presenta nuestra metodología de solución que está formada de cuatro fases: definición de la estructura, desarrollo del algoritmo, desarrollo de los módulos de navegación y pruebas (Figura 2), la información del contexto que va recolectándose en cada momento es almacenada en la base de datos.

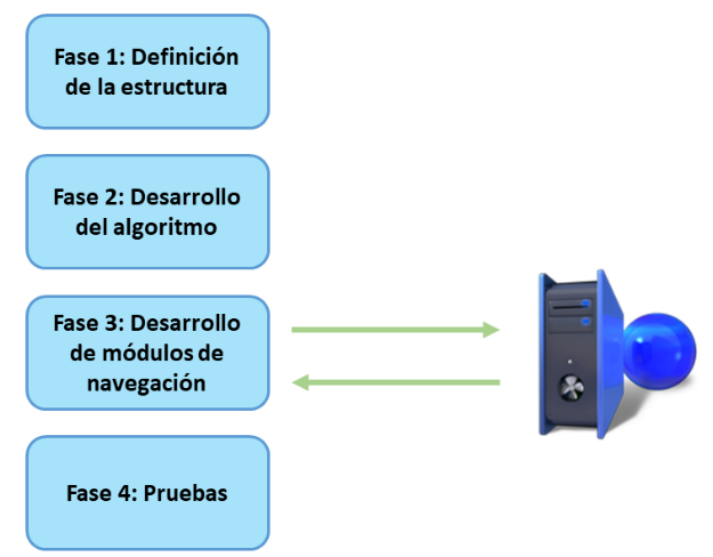

Fig. 1. Metodología de solución propuesta.

\subsection{Definición de estructura}

La primera fase de nuestra metodología de solución es la definición de la estructura, la cual tiene como objetivo registrar la organización en donde el sistema de navegación va a implementarse, así como los edificios de esta organización, el registro de los beacons que serán colocados en la organización y las rutas de navegación en cada uno de los edificios de la organización.

Para llevar a cabo la definición de la estructura fue necesario realizar un prototipo de software el cual se le denominó navIndoor, la cual es una aplicación móvil de Internet 
de las cosas para dispositivos móviles Android. La aplicación móvil tiene como objetivo gestionar los recursos que se necesitan para hacer una navegación en interiores.

La aplicación está destinada para ser usada por un usuario de la organización el cual le llamamos administrador de la organización, este usuario debe de tener los conocimientos del tipo de edificación en donde se hará la navegación de interiores, además de la información de los beacons que se utilizarán dentro de la organización.

La figura 3 muestra el menú de la aplicación navIndoor, la cual está formada de tres secciones: a)organizaciones, esta sección tiene como objetivo dar de alta, modificar o eliminar una organización, o un edificio dentro de la organización; b) gestión de beacons, esta sección permite al administrador de la organización capturar la información de cada uno de los beacons, estos son: valores uuid, major, minor, además de las credenciales que la compañía de los beacons brinda para poder ser utilizados; c) Rutas, en esta sección se registra las distancias existentes entre un beacon y otro, con esta información el algoritmo Dijkstra puede hacer el cálculo de la ruta óptima.
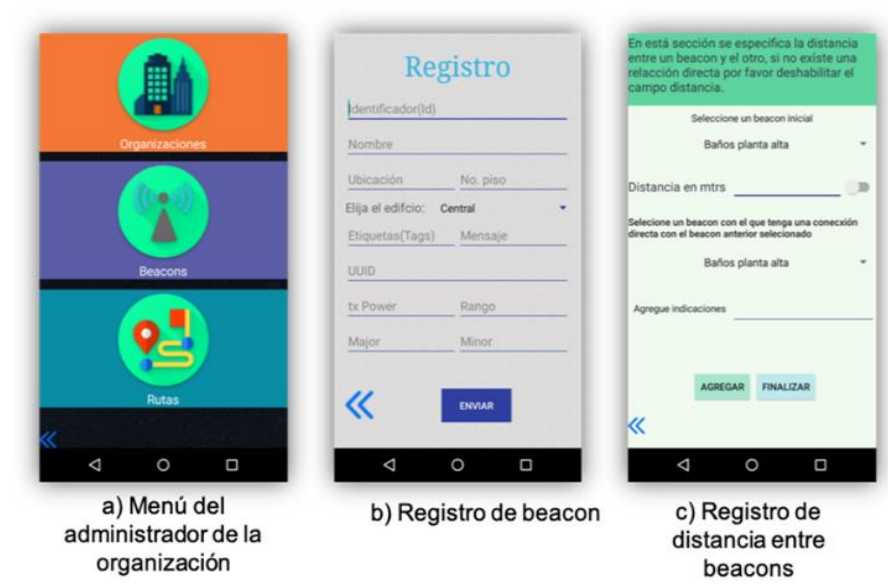

Fig. 2 Aplicación Móvil de IoT: navIndoor.

\subsection{Desarrollo de algoritmos de navegación}

La navegación de interiores que se propone en este artículo utiliza un grafo, el cual es una pareja de conjuntos $G=(V, A)$, donde $G$ es el grafo, $V$ es el conjunto de vértices o nodos, y $A$ es el conjunto de aristas que relacionan a los nodos, este último es un conjunto de pares de la forma $(u, v)$ tal que $u, v \varepsilon V[5]$. El algoritmo se genera por medio de una matriz de $n x n$ en la cual se establecen los valores de las distancias reales entre los beacons a usar, si es que existe un enlace directo, es decir que no se necesita pasar por un beacon intermedio, si no existe dicho enlace se establece un valor por default, esta matriz es generada por el administrador de la organización en la aplicación navIndoor.

Los algoritmos desarrollados para llevar a cabo la nevegación fueron un algoritmo de posicionamiento y un algoritmo de generación de rutas. Estos algoritmos juegan un rol muy importante en nuestra propuesta ya que mediante ellos se puedieron hacer las actividades mas importantes:

- Establecer la ubicación de un usuario, 
- Generar una ruta a partir de un punto inicial a un punto final.

Algoritmo de posicionamiento. Esté algoritmo tiene como objetivo obtener la ubicación de un usuario. Este algoritmo es utilizado para identificar el lugar donde se encuentra el usuario, es decir la ubicación del usuario aproximadamente, la cual nos ayudara saber cuál es su punto de partida, cual ha sido los lugares que ha estado, cuándo ha llegado a su destino.

Esta tarea se realiza con uso del SDK de los beacons Estimote, el cual nos proporciona una función de monitoreo que constante lanza un evento cuando algún usuario con su celular y la aplicación en ejecución entra dentro de la región de un beacon así podemos ejecutar cualquier actividad, como mostrar un mensaje, mostrar alguna imagen, hacer una consulta a la base de datos, etc.

Algoritmo de generación de rutas. esté algoritmo nos ayuda a poder formar las rutas a partir de la información generada por el administrador de la organización. Para poder generar una ruta es necesario tener un punto de inicio, un punto final y que exista al menos una distancia entre un beacon, es decir no debe de existir algún beacon que se encuentre aislado.

Existe una variedad de algoritmos que trabajan con rutas como puede ser: Kruskaly[15], Breadth first search[16], Bellman-Ford[17] los cuales trabajan de maneras diferentes por ejemplo Kruskaly trabaja con árboles de tipo de expansión mínima, Breadth first search y Bellman-Ford trabajan con grafos, pero las distancias (pesos en aristas) en caso de Breadth first search son distancias entre 0 y 1 y BellmanFord puede trabajar con cualquier tipo de distancias incluyendo negativas, sin embargo lo que necesitamos es que la información brindada por el administrador de la organización pase casi directamente y no hay un proceso de tratamiento de datos es por ello que se decidió utilizar el algoritmo Dijkstra además de trabajar con la ruta óptima. El algoritmo Dijkstra tuvo que ser modificado para poder hacer uso de él, de los cambios que se realizaron se agregó el parámetro del destino, se adaptaron los identificadores de los beacons como los nodos del grafo, se hizo con un número de nodos dinámico, se obtiene solo la ruta a la cual lleva al destino y finalmente se modificó para que trabajara con números de tipo decimal.

\subsection{Desarrollo de módulos de navegación}

El prototipo desarrollado en la tercera fase de la metodología propuesta se refiere al prototipo de navegación por parte de los usuarios, el cual está formado por cinco módulos los cuales fueron implementados en una aplicación móvil del sistema operativo Android. Este prototipo ha sido denominado como navIndoor:

- Registro de usuario,

- Inicio de sesión,

- Búsqueda de lugares dentro del edificio,

- Generación de ruta,

- Detección de ubicación.

navIndoor es una aplicación móvil de IoT para dispositivos móviles Android. Los usuarios deben registrarse para poder hacer uso de la aplicación desarrollada y poder hacer inicio de sesión. Una vez que el usuario se encuentra registrado e inicia sesión, 
entonces puede hacer búsquedas de los lugares dentro de un edificio inteligente, mostrándose la ubicación del usuario para posteriormente generar la ruta hacia del lugar al que el usuario desea ir.

Además, la aplicación navIndoor tiene la capacidad de poder detectar cuando no se ha seguido las instrucciones correctamente. La aplicación manda un mensaje con la descripción "Te has desviado de la ruta", posteriormente la aplicación automáticamente recalcula la ruta a partir de la posición actual del usuario. Otra funcionalidad que se incorporó a la aplicación es detectar si el usuario ha cambiado de edificio habiendo iniciado una ruta en un edificio diferente.

La Fig. 4 muestra una vista de la aplicación. La aplicación móvil tiene como objetivo guiar al usuario mediante una serie de instrucciones que la aplicación muestra y reproduce para llegar a un destino de interés en interiores.

En esta aplicación se hace uso del algoritmo de ubicación y del algoritmo Dijkstra, la aplicación detecta automáticamente en la organización que se encuentra, para posteriormente dar una ubicación más precisa mostrando en que edificio, en qué piso y en qué oficina, salón o baño se encuentra el usuario.

Posteriormente se busca el lugar donde se requiera ir, con esto se obtiene el punto inicial y punto final para poder ser enviados al algoritmo Dijkstra y obtener la ruta óptima, esta ruta se encuentra formada por al menos dos nodos los cuales tienen instrucciones que fueron dadas de altas por el administrador de la organización, finalmente se recorren todos estos nodos hasta llegar al final y haber concluido la navegación.

\subsection{Pruebas}

En esta se fase se enfocó en hacer pruebas al algoritmo Dijkstra para comprobar si se podía acoplar a las necesidades que teníamos. Se hicieron pruebas con la aplicación navIndoor con algoritmo Dijkstra y la interacción con los beacons. Se establecieron 3 pruebas con 3 diferentes grafos: 15 nodos; 20 nodos, y 30 nodos.

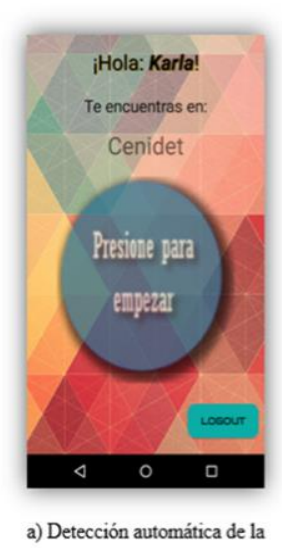
organización.
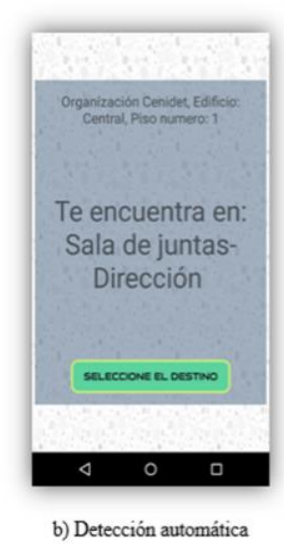

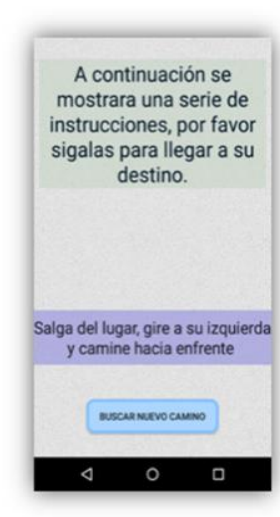

c) Recomendaciones a seguir

Fig. 3 Aplicación Móvil de IoT: navIndoor. 
En el grafo de 15 nodos se hicieron 8 recorridos teniendo una efectividad del $100 \%$ dando la ruta óptima en todos los recorridos.

En el grafo de 20 nodos se hicieron 10 recorridos teniendo una efectividad del $100 \%$ dando la ruta óptima en todos los recorridos.

En el grafo de 30 nodos se hicieron 15 recorridos teniendo una efectividad del $100 \%$ dando la ruta óptima en todos los recorridos.

En total se hicieron 33 recorridos desde los más sencillos recorriendo un solo nodo, hasta aquellos en donde se tuvieron que evaluar las distancias de los múltiples recorridos que existen para llegar al mismo destino.

En cuanto las pruebas de la aplicación navIndoor se hicieron tres diferentes pruebas con tres grafos elaborados a partir de un edifico de 2 pisos los grafos resultantes fueron los siguientes: 6 nodos; 4 nodos, y 10 nodos

En el grafo de 6 nodos se hicieron 5 recorridos teniendo una efectividad del $100 \%$ dando la ruta óptima en todos los recorridos.

En el grafo de 4 nodos se hicieron 3 recorridos teniendo una efectividad del $100 \%$ dando la ruta óptima en todos los recorridos.

En el grafo de 10 nodos se hicieron 2 recorridos teniendo una efectividad del $100 \%$ dando la ruta óptima en todos los recorridos.

Con los tres diferentes grafos la aplicación fue capaz de dar asesoramiento para llegar al destino seleccionado, además que siempre sugirió la ruta óptima.

\section{Conclusiones}

En este trabajo de investigación se presentó un sistema de navegación en interiores el cual permite dar una serie de indicaciones que el usuario tiene que seguir para llegar a su destino, las instrucciones que se dan al usuario permite recorrer la ruta óptima a partir de un punto inicial (ubicación del usuario) a un punto destino. Se demostró que la tecnológica beacon y el algoritmo Dijkstra es una opción viable para hacer navegación en interiores por los buenos resultados que se dieron. Se desarrolló una metodología de solución genérica, es decir que cualquiera que siga dicha metodología podrá hacer una navegación en interiores básica. La combinación de tecnologías como beacons, GPS y wifi podrían producir una herramienta muy poderosa de navegación en interiores haciendo más precisa la navegación, además de expandir dicha navegación a lugares abiertos dentro de las organizaciones sin tener que hacer un gasto fuerte en compra de beacons o de repetidores de señal.

Como trabajos futuros, puede explorarse la opción de mandar notificaciones a los usuarios cuando se haga una navegación en interiores en una organización nueva, Otra función que le daría un gran impulso sería que se hiciera una cartografía y geolocalización de la organización con sus edificios para poder ser mostrada la ruta y la ubicación del usuario gráficamente. Por otra parte, sería útil generar una versión web de la aplicación navIndoor para el administrador de la organización con el fin de brindarle un mejor espacio de trabajo.

Agradecimientos. Esta investigación ha sido financiada parcialmente por el proyecto SmartSDK, Num Ref. 272727, así como el proyecto TecNM/CENIDET, Num Ref. 5138.19-P. 


\section{Referencias}

1. Wagner, S., Fet, N., Handte, M., Marrón, P.J. An Approach for Hybrid Indoor/Outdoor Navigation. En: International Conference on Intelligent Environments (IE), pp.36-43 (2017)

2. Bouskela, M., Casseb, M., Bassi, S., De Luca, C., Facchina, M. La ruta hacia las Smart Cities. Migrando de una gestión tradicional a la ciudad inteligente. Biblioteca Felipe Herrera Del Banco Interamericano de Desarrollo, pp. 454 (2016)

3. Cisco, https://newsroom.cisco.com/feature-content?articleId=1208342, último acceso: 02/05/2019

4. Giménez Rodríguez T.: Sistema de Posicionamiento Global (GPS): Sociedad Latinoamericana en Percepción Remota y Sistemas de Información Espacial., pp. 94 (2008)

5. infSOfT, https://www.infsoft.com/solutions/indoor-navigation, último acceso: 01/05/2019

6. Shende, P., Mehendarge, S., Chougule, S., Kulkarni, P., Hatwar, U.: Innovative ideas to improve shopping mall experience over E-commerce websites using beacon technology and data mining algorithms. En: International Conference on Circuit, Power and Computing Technologies, pp. 1-5 (2017)

7. What is UUID: https://searchmicroservices.techtarget.com/definition/UUID-UniversalUnique-Identifier/, último acceso: 20/12/2018.

8. Beacon Strategy Guide - UUID, Major, Minor: https://kontakt.io/blog/beacon-id-strategyguide-quick-deployment/, último acceso: 20/12/2018.

9. Chawathe, S.: Beacon Placement for Indoor Localization using Bluetooth. En: 11th International IEEE Conference on Intelligent Transportation Systems, pp. 980-985 (2008)

10. Chen, Z., Zhu, Q., Jiang, H., Soh, Y.C.: Indoor localization using smartphone sensors and iBeacons. En: IEEE 10th Conference on Industrial Electronics and Applications, pp. 1723$1728(2015)$

11. Ozdenizci, B., Ok, K., Coskun, V., Aydin, M.N.: Development of an Indoor Navigation System Using NFC Technology. En: Fourth International Conference on Information and Computing, pp. 11-14 (2011)

12. Ingresa a los edificios con Mapas de interiores: https://www.google.com/intl/es419_mx/maps/about/partners/indoormaps/, último acceso: 01/05/2019

13. Kasim, S.: Indoor Navigation Using A* Algorithm. En: Herawan T., Ghazali R., Nawi N., Deris M. (eds) Recent Advances on Soft Computing and Data Mining. SCDM 2016. Advances in Intelligent Systems and Computing, vol 549 (2017)

14. Kruskal's Minimum Spanning Tree Algorithm | Greedy Algo-2. https://www.geeksforgeeks.org/kruskals-minimum-spanning-tree-algorithm-greedy-algo-2/, último acceso: 20/12/2018

15. The breadth-first search algorithm: https://www.khanacademy.org/computing/computerscience/algorithms/breadth-first-search/a/the-breadth-first-search-algorithm/, último acceso: 20/12/2018

16. Goldberg, A., Radzik, T.A.: Heuristic improvement of the Bellman-Ford algorithm. Stanford Univ CA Dept. of Computer Science (1993) 